\title{
The Role of MIG/CXCL9 in Cardiac Allograft Vasculopathy
}

\author{
James J. Yun, ${ }^{* \dagger}$ Michael P. Fischbein, ${ }^{*}$ \\ David Whiting, ${ }^{*}$ Yoshihito Irie, ${ }^{*}$ \\ Michael C. Fishbein, ${ }^{\dagger}$ Marie D. Burdick, ${ }^{\ddagger}$ \\ John Belperio, ${ }^{\ddagger}$ Robert M. Strieter, ${ }^{\ddagger}$ Hillel Laks, ${ }^{*}$ \\ Judith A. Berliner, ${ }^{\dagger}$ and Abbas Ardehali* \\ From the Division of Cardiothoracic Surgery," Department of \\ Surgery, and the Departments of Pathology and Laboratory \\ Medicine ${ }^{\dagger}$ and Medicine, ${ }^{\ddagger}$ University of California at Los Angeles, \\ Los Angeles, California
}

T lymphocytes play a critical role in chronic rejection of transplanted hearts, or cardiac allograft vasculopathy (CAV). However, the molecular mediators of $T$ lymphocyte recruitment in CAV are incompletely defined. We hypothesized that the chemokine, monokine induced by interferon- $\gamma$ (MIG/CXCL9), which induces $T$ lymphocyte migration in vitro, participates in $T$ lymphocyte recruitment in CAV. In a previously characterized MHC II-mismatched murine model of CAV, intragraft MIG/CXCL9 gene transcript and protein levels increased on days 7, 14, and 24 days after transplantation, paralleling $T$ lymphocyte recruitment and preceding intimal thickening. Antibody neutralization of MIG/CXCL9 significantly reduced $\mathrm{CD}^{+} \mathrm{T}$ lymphocyte infiltration and intimal thickening in this model. MIG/CXCL9 was produced by graftinfiltrating MOMA-2+ macrophages in early and late stages of CAV. And, although T lymphocytes did not produce MIG/CXCL9, recipient $\mathrm{CD}^{+} \mathrm{T}$ lymphocytes were required for sustained intragraft MIG/CXCL9 production and CAV development. These findings demonstrate that 1) MIG/CXCL9 plays an important role in $\mathrm{CD}^{+} \mathrm{T}$ lymphocyte recruitment and development of CAV , 2) MOMA-2+ macrophages are the predominant recipient-derived source of MIG/CXCL9, and 3) recipient $\mathrm{CD} 4$ lymphocytes are necessary for sustained MIG/CXCL9 production and CAV development in this model. Neutralization of the chemokine MIG/CXCL9 may have therapeutic potential for the treatment of chronic rejection after heart transplantation. (Am J Patbol 2002, 161:1307-1313)

Chronic rejection of transplanted organs remains the major barrier to the long-term success of clinical transplantation. In transplanted hearts, the primary manifestation of chronic rejection is cardiac allograft vasculopathy (CAV). CAV is characterized by diffuse, concentric intimal thick- ening of the donor heart arteries and veins, and is currently the leading cause of late allograft failure. There has been limited success with pharmacological therapy or revascularization procedures in this disorder. ${ }^{1}$

Both alloantigen-dependent and -independent factors have been shown to contribute to the development of CAV. ${ }^{2}$ However, the host immune responses to the disparate alloantigens are the dominant factor in the development of CAV. Both CD4 and CD8 lymphocytes participate in the initiation and progression of lesion development. $^{3,4}$ Despite their well-known causal role in $\mathrm{CAV}$, the molecular mechanisms that mediate T lymphocyte recruitment into the graft are not fully defined.

Chemokines, a subfamily of cytokines, induce the directional migration of $\mathrm{T}$ lymphocytes and other mononuclear cells in vitro and in vivo. ${ }^{5}$ Reports from other laboratories and our group have demonstrated up-regulation of various chemokines correlating with $T$ lymphocyte recruitment, during acute and chronic rejection of allografts. 6,7 The interferon (IFN)- $\gamma$-inducible CXC chemokine, monokine induced by IFN- $\gamma$ (MIG/CXCL9), is a potent chemotactic factor for $\mathrm{T}$ lymphocytes, and has been shown to contribute to a variety of inflammatory disorders. ${ }^{8-10}$ The importance of MIG/CXCL9 in acute rejection of allografts has been documented. ${ }^{11,12}$ Miura and colleagues $^{12}$ demonstrated that MIG/CXCL9 is a dominant factor in recruitment of $\mathrm{T}$ lymphocytes in acute rejection of MHC-mismatched cardiac allografts. In contrast, the role of MIG/CXCL9 in CAV remains unknown.

In a previously characterized $\mathrm{MHC}$ II-mismatched murine model of CAV, we have observed a temporal correlation between intragraft IFN- $\gamma$ level and mononuclear cell recruitment. We hypothesized that intragraft IFN- $\gamma$ induces up-regulation of MIG/CXCL9, which in turn may mediate the $\mathrm{T}$ lymphocyte recruitment in this model of CAV. First, we sought to define the time course of intragraft MIG/CXCL9 production in relation to T lymphocyte recruitment and CAV development. Second, with a neutralizing antibody to MIG/CXCL9, we aimed to determine the functional role of MIG/CXCL9 on T Iymphocyte recruit-

Supported by funds from the American Association for Thoracic Surgery (Second Alfred Blalock Award to A. A.), the American Heart Association (0130105N to A. A.), the Piansky Family Trust (to M. C. F.), and the National Institutes of Health (PO1 HL30568 to J. A. B., T32 AI07126-23 to J. J. Y., and P01 HL67665 to R. M. S.).

Accepted for publication June 28, 2002.

Address reprint requests to Abbas Ardehali, M.D., Division of Cardiothoracic Surgery, 62-246 CHS, UCLA Medical Center, 10833 Le Conte Ave., Los Angeles, CA 90095. E-mail: aardehali@mednet.ucla.edu. 
ment and CAV development in this MHC II-mismatched model of CAV. We also sought to determine which graftinfiltrating cells produced MIG/CXCL9 in this model. And lastly, we investigated the relationship between recipient CD4 lymphocytes and intragraft MIG/CXCL9 level.

\section{Materials and Methods}

\section{Animals}

Adult female B6C.H-2 $2^{\text {bm12 }}$, wild-type (WT) C57BL/6, and CD4 knockout (KO) (on C57BL/6 background) mice (7 to 10 weeks old) were purchased from the Jackson Laboratories (Bar Harbor, ME). The B6C.H-2 ${ }^{\mathrm{bm} 12}$ and C57BL/6 strains differ at the I-A locus of MHC II but are identical at $\mathrm{MHC}$ I and minor MHC loci. The mice were housed under conventional conditions and fed water and standard rodent laboratory chow (Purina Mills, Inc., St. Louis, MO) ad libitum. All animals received humane care in compliance with the University of California at Los Angeles guidelines and the Principles of Laboratory Animal Care published by the National Institutes of Health (NIH Publication No. 8623, revised 1985).

\section{Transplantation}

Intra-abdominal heterotopic cardiac transplantation was performed using a modification of the method outlined by Corry and colleagues. ${ }^{13}$ Donor hearts were arrested with heparinized saline (100 U/1cc), excised, and stored on ice. Through a midline abdominal incision, the donor aorta was anastamosed to the recipient infrarenal abdominal aorta, and the donor pulmonary artery was anastamosed to the inferior vena cava with 10-0 nylon suture. Ischemia time was $60 \pm 15$ minutes. The transplanted hearts spontaneously resumed contractions after reperfusion.

All animals alive on postoperative day 1 , survived to the postoperative day 24. All of the donor hearts had palpable contractions on day 24 after transplantation

\section{Experimental Groups}

B6C.H-2 $2^{\mathrm{bm} 12}$ strain mouse hearts were transplanted into WT C57BL/6 (allografts) or CD4 KO recipient mice; the donor hearts were harvested on days $4,7,14$, or 24 after transplant ( $n=6$ per time point). C57BL/6 mouse donor hearts were also transplanted into WT C57BL/6 recipient mice (isografts), which were sacrificed at the same time points ( $n=4$ per time point). Donor hearts that were removed but not transplanted (day 0) were also used as controls. Each animal was analyzed separately as a single experiment.

A separate group of allograft recipients were treated with $0.5 \mathrm{ml}$ of polyclonal goat anti-mouse MIG/CXCL9 antibody (see below) $(n=4)$ or control antibody $(n=4)$ intraperitoneally on postoperative days $5,7,9,11,13,15$, 17 , and 19 before sacrifice on postoperative day 24 . No immunosuppression was given.
Table 1. Chemotaxis of IL-2-Stimulated CXCR3-Expressing Splenocytes

\begin{tabular}{lcc}
\hline & $\begin{array}{c}\text { Control } \\
\text { antibody }\end{array}$ & $\begin{array}{c}\text { Anti-MIG/ } \\
\text { CXCL9 } \\
\text { antibody }\end{array}$ \\
\hline Murine MIG/CXCL9 & $101.3 \pm 9$ & $46.1 \pm 4$ \\
Murine IP-10/CXCL10 & $105.2 \pm 12$ & $94.4 \pm 7$ \\
Murine SDF-I $\beta$ & $183.7 \pm 14$ & $198.4 \pm 17$ \\
Murine RANTES/CCL5 & $74.4 \pm 6$ & $87.1 \pm 7$ \\
Control vehicle & $6.1 \pm 2$ & $7.8 \pm 4$ \\
\hline
\end{tabular}

$P<.05$ when compared to control antibody.

Anti-MIG/CXCL9 demonstrated specific neutralization of murine MIG/CXCL9 and inhibition of chemotaxis.

\section{Anti-MIG/CXCL9 Preparation and Analysis}

Polyclonal goat anti-mouse MIG/CXCL9 antibody was produced by immunization of goats to purified recombinant murine MIG/CXCL9 (R\&D Systems, Minneapolis, MN). The serum was used for specificity analyses and neutralization when antisera titers had reached greater than $1 / 1,000,000$ by direct enzyme-linked immunosorbent assay. The specificity of anti-MIG/CXCL9 antiserum was confirmed in our sandwich enzyme-linked immunosorbent assay without cross-reactivity to a panel of murine cytokines [interleukin (IL)-1R $\alpha, \mathrm{IL}-1, \mathrm{IL}-2, \mathrm{IL}-6$, tumor necrosis factor- $\alpha$, and IFN- $\gamma$ ] and murine CXC and $\mathrm{CC}$ chemokines. Furthermore, the specificity of anti-MIG/ CXCL9 antisera was also ascertained by chemotaxis assay. Briefly, murine splenocytes were stimulated with IL-2 for 10 days and their CXCR3 expression was confirmed by fluorescence-activated cell sorting (FACS). Fifty $\mathrm{ng} / \mathrm{ml}$ of murine MIG/CXCL9, IP-10/CXCL10, SDF-1 $\beta / C X C L 12$, or RANTES/CCL5 were preincubated with anti-MIG/ CXCL9 antibody or control antibody for 30 minutes. Chemotaxis assay was performed with 5.0- $\mu \mathrm{m}$ polycarbonate filters coated with fibronectin (ICN Biomedicals, Irvine, CA) as previously described. ${ }^{14}$ The anti-MIG/CXCL9 antibody demonstrated specific neutralizing capacity of murine MIG/CXCL9; it inhibited splenocyte chemotaxis response to murine MIG/CXCL9. In contrast, anti-MIG/ CXCL9 antibody had no cross-reactivity with murine IP10/CXCL10, murine SDF-1 $\beta / C X C L 12$, or murine RANTES/ CCL5 (Table 1).

The antibody for intracellular staining was a goat polyclonal anti-mouse MIG/CXCL9 antibody and was obtained from R\&D Systems. This antibody shows less than $5 \%$ cross-reactivity with rmMIP-2, rmRANTES, rhIP-10, and a panel of other murine chemokines.

\section{Ribonuclease Protection Assay}

Total cellular RNA was isolated from apical ventricular tissue using the Trizol method (Life Technologies, Gaithersburg, MD) according to the manufacturer's directions. RNA quality was confirmed with gel electrophoresis. MIG/ CXCL9 gene expression was determined using an RNase protection assay (Pharmingen, San Diego, CA) as previously described.$^{15}$ Briefly, a ${ }^{32}$ P-UTP-labeled probe complementary to chemokine sequences of MIG/CXCL9 was synthesized in vitro and hybridized to $15 \mu \mathrm{g}$ of sample 
RNA for 16 hours. Reaction mixtures then underwent RNase and proteinase treatments to degrade nonhybridized species. Final reaction products were electrophoresed on a 5\% polyacrylamide gel. Autoradiography was performed for 16 hours at $-80^{\circ} \mathrm{C}$. A phosphorimager was used to quantitate autoradiograph band intensity and phosphorimager measurements were normalized to the GAPDH signal within the same sample lane.

\section{Protein Isolation and Enzyme-Linked Immunosorbent Assay}

Basal sections from transplanted hearts were placed in phosphate-buffered saline containing $1 \mathrm{mmol} / \mathrm{L}$ phenylmethyl sulfonyl fluoride, $1 \mathrm{mmol} / \mathrm{L}$ ethylenediaminetetraacetic acid, $1 \mu \mathrm{g} / \mathrm{ml}$ leupeptin, $1 \mu \mathrm{g} / \mathrm{ml}$ pepstatin, and $1 \mu \mathrm{g} / \mathrm{ml}$ aprotinin (all from Sigma, St. Louis, MO) before homogenization with a T25 Ultra-Turrax 10-mm dispersion element (IKA Works; Jepson-Bolton, Watford Herts, UK). Samples were centrifuged at $15,000 \times g$ for 15 minutes and the soluble fraction retained for analysis. Intragraft IFN- $\gamma$ protein levels were measured with an enzyme-linked immunosorbent assay (R\&D Systems) according to the manufacturer's instructions. Intragraft MIG/ CXCL9 protein levels were measured with an enzymelinked immunosorbent assay as previously described. ${ }^{14}$

\section{Morphometric Analysis}

Elastica von Gieson stains were performed on transverse sections of allografts and isografts. Vessel size was measured with computer-based software (Optimas, Seattle, WA); only vessels exceeding $80 \mu \mathrm{m}$ in diameter were included. For control and anti-MIG/CXCL9-treated groups, $>30$ vessels were analyzed. Luminal $(L)$ and intimal and luminal $(\mathrm{I}+\mathrm{L})$ areas were traced, and areas were quantitated with the Optimas program. Intimal thickening (\% stenosis) was calculated according to the formula: intimal thickening $=\mathrm{I} / \mathrm{I}+\mathrm{L}$ and expressed as a percentage.

\section{Graft-Infiltrating Cell Isolation and FACS Analysis}

Hearts were minced and digested for 2 hours in collagenase D (2 mg/ml; Worthington Biochemical, Lakewood, $\mathrm{NJ}$ ) at room temperature in RPMI 1640 media (RPMI) with $10 \%$ fetal calf serum (Life Technologies, Inc., Rockville, MD). Isolated cells were collected, washed twice in RPMI and $5 \%$ fetal calf serum, and counted after lysis of erythrocytes. Cells were subsequently incubated with fluorochrome-conjugated antibodies specific for cell surface antigens (rat anti-mouse CD4, CD8, and MOMA-2 antibodies; Pharmingen, San Diego, CA, and Serotec USA, Raleigh, NC) for 30 minutes at $4^{\circ} \mathrm{C}$. FACS analysis of labeled cells was performed with a single-laser argon flow cytometer (Becton Dickinson, Franklin Lakes, NJ). The net percentage of cells positive for the cell surface markers CD4, CD8, or MOMA-2 was calculated by subtracting the background staining (percentage of fluoro-

\section{Intragraft IFN-gamma Levels}

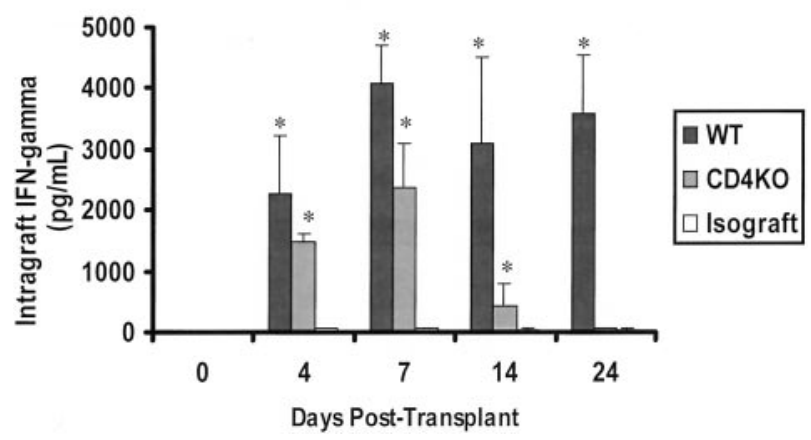

Figure 1. Intragraft IFN- $\gamma$ level increased in allografts starting at day 4 after transplantation and remained elevated at the other examined time points. There was a transient rise in IFN- $\gamma$ level in the donor hearts transplanted into $\mathrm{CD} 4-/-$ recipients. IFN- $\gamma$ level in isografts was minimal $(n=6$ per time point).

chrome-positive cells labeled with irrelevant isotypematched control antibody) from the total staining (percentage of fluorochrome-positive cells labeled with anti-CD4, CD8, or MOMA-2 antibody). The number of fluorochrome-positive cells is reported as the number of fluorochrome-positive cells per gram of tissue. The intracellular chemokine assay was performed as previously reported. ${ }^{15}$

\section{Histology and Immunohistochemistry}

Frozen sections $(4 \mu \mathrm{m})$ of transplanted mouse hearts were either stained with hematoxylin and eosin or immunostained for CD4 or CD8 with standard avidin-biotin technique (Vectastain ABC; Vector Laboratories, Burlingame, CA). Controls included omission of primary antibody and use of irrelevant isotype-matched primary antibody.

\section{Statistics}

Data are presented as mean \pm SEM. Analysis of variance was used to compare mean values of cell number, protein levels, and intimal thickening between different time points. A value of $P<0.05$ was considered significant.

\section{Results}

\section{Time Course of IFN- $\gamma$ Production}

Intragraft IFN- $\gamma$ protein levels were low or undetectable in day 0 hearts (control), but increased to $2257 \pm 960 \mathrm{pg} / \mathrm{ml}$ in the allografts by day 4 (Figure 1). On days 7, 14, and 24 after transplantation, the intragraft IFN- $\gamma$ protein levels increased further to $\geq 3000 \mathrm{pg} / \mathrm{ml}$. In contrast, intragraft IFN- $\gamma$ protein levels in isografts remained $\leq 56 \mathrm{pg} / \mathrm{ml}$ at all time points examined. 


\section{A Allograft Isograft Days Post-Transplant: $\begin{array}{lllllll}0 & 7 & 14 & 24 & 7 & 14 & 24\end{array}$}
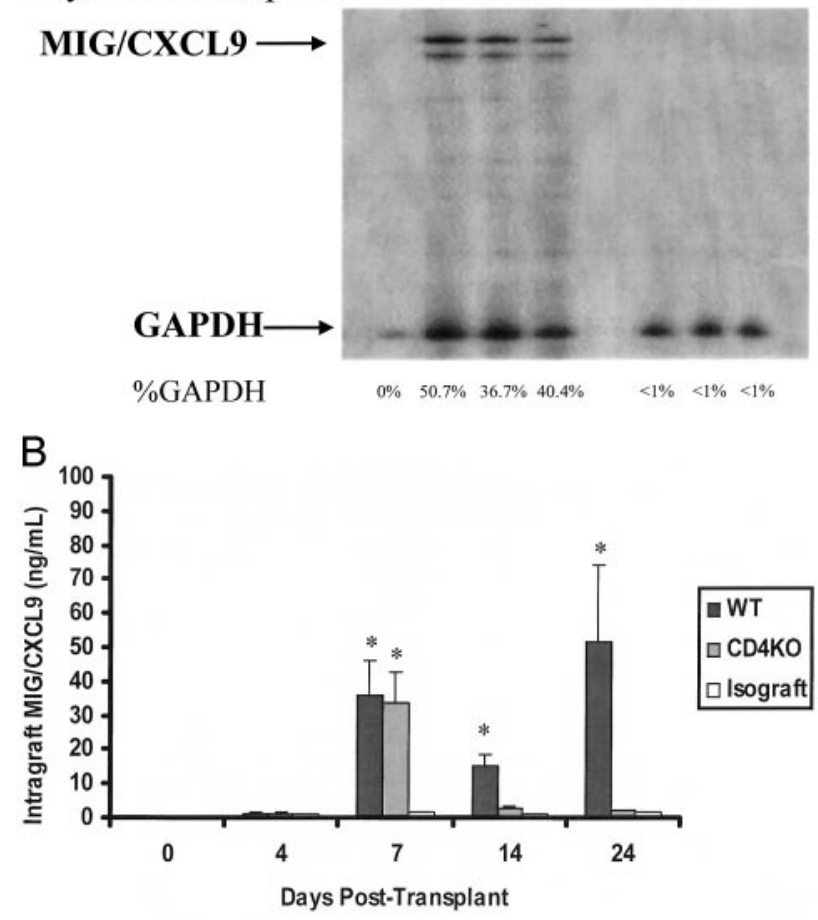

${ }^{*} \mathrm{p}<0.01$ vs. isograft

Figure 2. A: Representative autoradiographs showing the temporal evoluFigure 2. A: Representative autoradiographs showing the temporal evolu-
tion of MIG/CXCL9 gene expression in allografts and isografts. Day 0 hearts were harvested but not transplanted; they showed no MIG/CXCL9 gene expression. In allografts, MIG/CXCL9 gene expression was detected at day 7 and persisted through day 24. In isografts, no MIG/CXCL9 gene up-regulation was detected. B: Intragraft MIG/CXCL9 protein level in the allografts increased on day 7 after transplantation and remained elevated on days 14 and 24. There was a transient rise in intragraft MIG/CXCL9 levels in the donor hearts in CD4 KO recipients; intragraft MIG/CXCL9 levels at days 14 and 24 decreased significantly. This pattern of MIG/CXCL9 expression correlated with IFN- $\gamma$ levels in the donor hearts transplanted into $\mathrm{CD} 4-/-$ recipients, in a delayed manner (See Figure 1). There was minimal MIG/CXCL9 production in isografts at all examined time points ( $n=6$ per time point).

\section{Intragraft MIG/CXCL9 Production Correlates} With T Lymphocyte Recruitment and Precedes CAV Development

MIG/CXCL9 gene transcript levels were present in the allografts harvested at days 7, 14, and 24 after transplantation (Figure 2A). MIG/CXCL9 protein levels paralleled MIG/CXCL9 gene expression (Figure 2B). By day 7, MIG/ CXCL9 protein levels in allografts increased significantly to $36 \pm 10 \mathrm{ng} / \mathrm{ml}(P<0.001$ versus day 0$)$, and remained elevated at days 14 and 24. Notably, intragraft MIG/CXCL9 levels were low or undetectable at day 4 (Figure 2B).

In this model of CAV, high intragraft MIG/CXCL9 protein levels correlated with mononuclear cell recruitment. Infiltration of $\mathrm{CD}^{+}$and $\mathrm{CD} 8+\mathrm{T}$ lymphocytes increased at both days 7 and 14 (Figure 3). By day 14, T lymphocytes and macrophages had localized to the perivascular areas as reported previously. ${ }^{7}$ Intragraft MIG/CXCL9 production and mononuclear cell recruitment preceded development of intimal thickening, which was detectable by

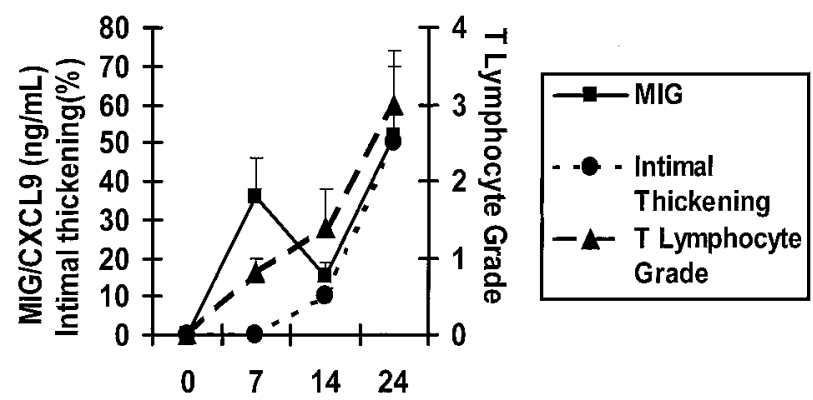

\section{Days Post-Transplant}

Figure 3. Line graph showing time course of intragraft MIG/CXCL9 protein level, T lymphocyte grade, and intimal proliferation. Increased MIG/CXCL9 production (day 7) paralleled initial T lymphocyte recruitment and preceded increased intimal thickening (day 14) ( $n=6$ per time point).

day $14(10 \pm 2 \%)$ and increased significantly by day 24 $(50 \pm 7 \%)$.

In isografts, MIG/CXCL9 gene expression was not detected (Figure 2A). Intragraft MIG/CXCL9 protein levels were low or undetectable at all time points ( $>40$-fold less than allografts; Figure 2B). Furthermore, the isografts displayed no mononuclear cell infiltration at 7, 14, and 24 days and lacked intimal thickening (data not shown).

\section{MIG/CXCL9 Neutralization Attenuates CD4+ $T$ Lymphocyte Recruitment and CAV Development}

Because intimal thickening is the principal manifestation of CAV in humans, we analyzed the severity of intimal thickening in hearts transplanted into anti-MIG/CXCL9 antibody-treated or control serum-treated mice at day 24. Hearts transplanted into anti-MIG/CXCL9 antibodytreated mice had significantly less intimal thickening (20 $\pm 5 \%$, Figure 4) than hearts transplanted into control antibody treated mice $(52 \pm 5 \%, P<0.05$ versus antiMIG/CXCL9; Figure 4).

To delineate the mechanism by which anti-MIG/CXCL9 antibody treatment attenuated CAV, we analyzed transplanted hearts from mice treated with anti-MIG/CXCL9 antibody or control antibody. Analysis of graft-infiltrating cells on day 24 revealed a marked decline in the number of $\mathrm{CD}^{+}{ }^{+} \mathrm{T}$ lymphocytes in anti-MIG/CXCL9 antibodytreated recipients. FACS analysis revealed that the number of intragraft $\mathrm{CD} 4^{+} \mathrm{T}$ lymphocytes were decreased sixfold by anti-MIG/CXCL9 serum (Figure 5). No change in CD8 lymphocyte infiltration was observed on day 24 after transplantation. Anti-MIG/CXCL9 antibody did not affect the numbers of peripheral lymphoid $\mathrm{CD} 4^{+} \mathrm{T}$ lymphocytes, CD8+ T lymphocytes, or MOMA-2+ macrophages (data not shown).

\section{MOMA-2+ Macrophages Produce MIG/CXCL9 in Early and Late CAV}

To identify the cellular sources of MIG/CXCL9, we performed FACS on graft-infiltrating cells stained for the cell 


\section{Anti-MIG/CXCL9 Antibody Control Antibody}

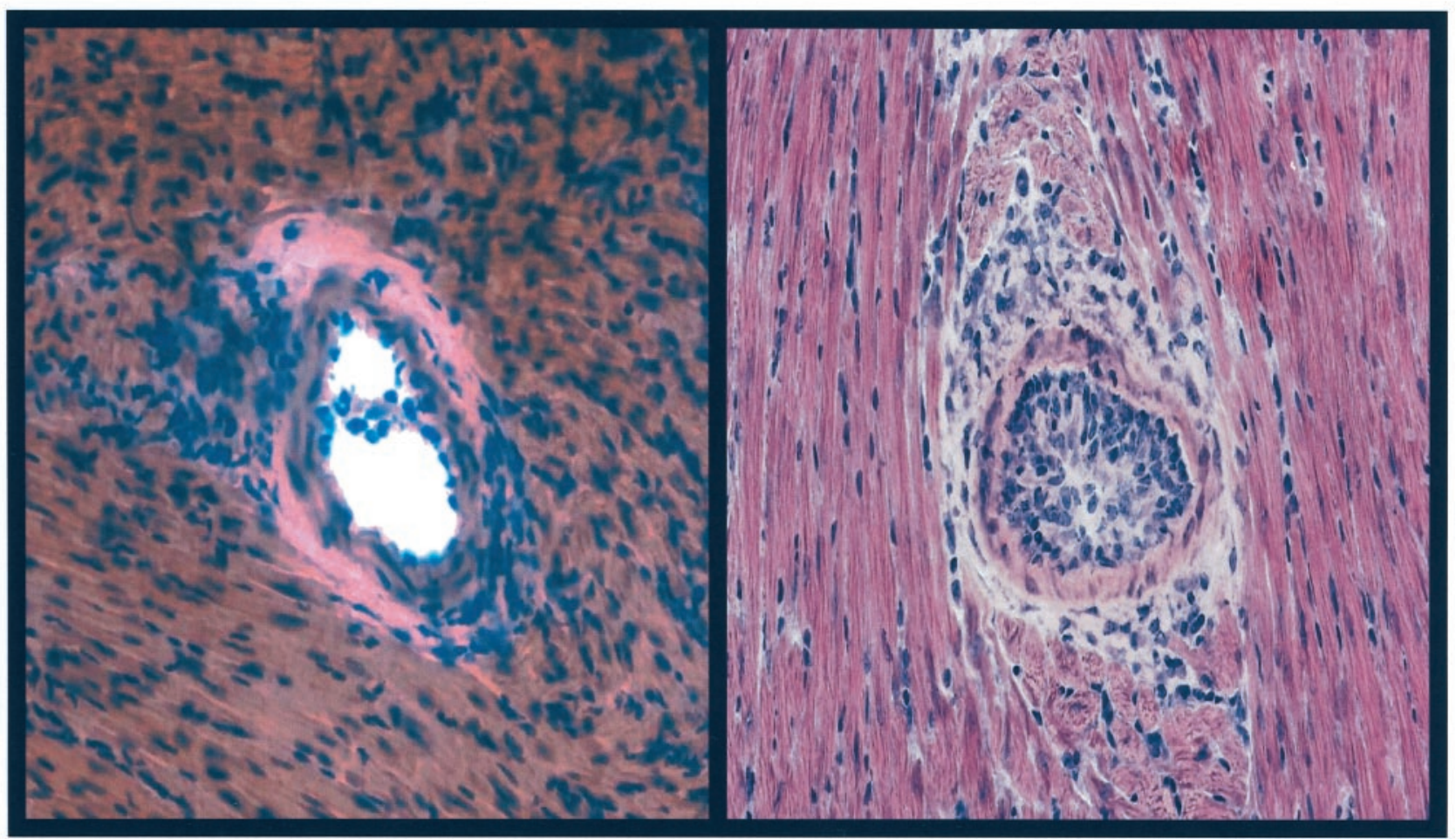

Figure 4. MIG/CXCL9 neutralization attenuates development of intimal thickening. Representative histology of anti-MIG/CXCL9 antibody-treated allografts and control antibody-treated allografts at 24 days after transplant (H\&E; original magnifications, $\times 100)$.

surface markers (CD4, CD8, MOMA-2, natural killer), and intracellular MIG/CXCL9 protein (with a phycoerythrinconjugated anti-murine MIG/CXCL9 antibody).

At day 7 , intracellular MIG/CXCL9 was detected by FACS in $10 \pm 2 \times 10^{5}$ MOMA-2+ macrophages in allografts. By day 24, the number of MIG/CXCL9producing MOMA-2+ increased more than eightfold (Figure 6). Despite the substantial rise in the number of MIG/CXCL9-producing macrophages, the total intra-

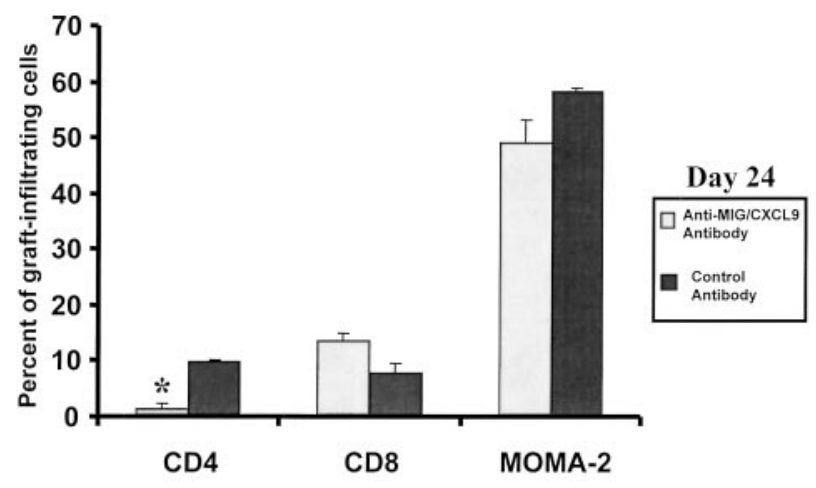

Figure 5. Graft-infiltrating cells were quantitated in the donor hearts transplanted into anti-MIG/CXCL9 or control antibody-treated recipients on day 24 after transplantation. Treatment with anti-MIG/CXCL9 antibody resulted in a significant reduction in the number of graft-infiltrating CD4 lymphocytes, but did not affect the number of intragraft CD8 lymphocytes or MOMA-2+ cells $(n=4)$. graft MIG/CXCL9 level did not change between days 7 and 24. This observation suggests that the amount of MIG/CXCL9 produced by each MOMA-2+ macrophage had decreased at 24 days, when compared to day 7 after transplantation.

MIG/CXCL9 was not detected in $\mathrm{CD} 4^{+} / \mathrm{CD} 8+\mathrm{T}$ lymphocytes or natural killer cells. No MIG/CXCL9-producing graft-infiltrating cells were identified in isografts (data not shown).

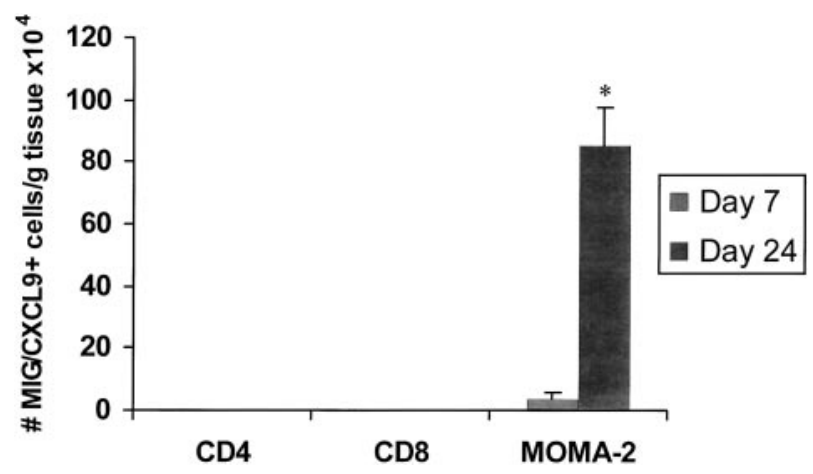

Figure 6. FACS analysis of cells isolated at days 7 and 24 from allografts. The number of cells staining positively for MIG/CXCL9 and also expressing the cell surface markers CD4, CD8, and MOMA-2+ (macrophage), are shown ( $n=4$ hearts analyzed per time point). MIG/CXCL9 production was detected in MOMA-2+ macrophages, but not in CD4 or CD8 T lymphocytes. The number of MIG/CXCL9-producing MOMA-2+ macrophages increased by more than 10-fold between days 7 and 24 . 


\section{Recipient $C D 4^{+}$Lymphocytes Are Required for Sustained MIG/CXCL9 Protein Production}

We have previously shown that in this MHC class IImismatched model, CAV development is dependent on CD4 lymphocytes; the allografts in CD4 KO recipients do not develop CAV. ${ }^{16}$ To determine the role of CD4 lymphocytes in intragraft MIG/CXCL9 production, we assayed both IFN- $\gamma$ and MIG/CXCL9 levels in the donor hearts transplanted into CD4 KO recipients. IFN- $\gamma$ was detectable on day 4 and peaked on day 7 after transplantation, in the donor hearts transplanted into CD4 KO recipients. Thereafter, the level declined. The pattern of MIG/CXCL9 production paralleled the IFN- $\gamma$ level, but was slightly delayed. There was a transient rise in MIG/ CXCL9 level to $2836 \pm 642 \mathrm{pg} / \mathrm{ml}$ at day 7 in the donor hearts transplanted into CD4 KO recipients (Figure 2B). Intragraft MIG/CXCL9 levels at days 14 and 24 declined significantly to $<1000 \mathrm{pg} / \mathrm{ml}(P<0.001$ versus day 7$)$, and were significantly lower $(P<0.05)$ than those in donor hearts transplanted into WT recipients at all examined time points (Figure 2B). At 7 days, the allografts in CD4 KO recipients lacked CD4 and natural killer cells entirely and contained threefold fewer CD8 lymphocytes and MOMA-2+ macrophages, when compared to allografts in WT mice (data not shown). By day 24, the allografts in $\mathrm{CD} 4 \mathrm{KO}$ recipients lacked any mononuclear cell infiltrate and as expected, did not display any intimal lesions.

\section{Discussion}

This study demonstrates that 1) in a MHC II-mismatched murine model of CAV, there is a significant and sustained up-regulation of MIG/CXCL9 (at both the gene transcript and protein level) that correlates with $\mathrm{T}$ lymphocyte recruitment and precedes intimal thickening, 2) neutralization of MIG/CXCL9 significantly reduces CD4 lymphocyte recruitment and intimal thickening, 3) MOMA-2+ macrophages are the predominant recipient-derived cells that produce MIG/CXCL9, and 4) sustained MIG/CXCL9 production in this model is dependent on recipient CD4 lymphocytes.

IFN- $\gamma$ has been shown to be pivotal in rejection of skin grafts in this MHC II-mismatched model. ${ }^{17}$ It has been suggested that IFN- $\gamma$ up-regulates MHC class II antigens and hence accentuates graft immunogenicity. More recently, Koga and colleagues ${ }^{11}$ have elegantly demonstrated the role of IFN- $\gamma$ via induction of MIG/CXCL9 in acute rejection of skin grafts in this model. IFN- $\gamma$-deficient recipients were not able to reject $\mathrm{B} 6 \mathrm{C} . \mathrm{H}-2^{\mathrm{bm} 12}$ grafts; injection of MIG/CXCL9 into the graft restored T Iymphocyte infiltration and rejection. Furthermore, neutralization of MIG/CXCL9 inhibited T lymphocyte infiltration and late rejection of skin grafts in WT recipients.

The findings of our study also substantiate a critical role for MIG/CXCL9 in T lymphocyte recruitment in chronic rejection by demonstrating 1) significant up-regulation of MIG/CXCL9 in allografts, but not isografts; 2) a temporal correlation between MIG/CXCL9 up-regulation and T lymphocyte recruitment, preceding CAV development; and 3) attenuation of CAV by neutralization of MIG/ CXCL9. However, unlike rejection of skin grafts in this MHC class II mismatched model, neutralization of MIG/ CXCL9 did not completely abrogate intragraft T lymphocyte recruitment and intimal lesion development. One possibility is that neutralization of MIG/CXCL9 in this model was incomplete. Analyses of two donor hearts in recipients treated with anti-MIG/CXCL9 antibody at each time point revealed undetectable MIG/CXCL9 level, suggesting that the administered regimen is sufficient to neutralize MIG/CXCL9 in this model. Another possibility is that $T$ lymphocyte recruitment into cardiac allografts in this model, unlike skin grafts, is mediated by MIG/CXCL9 as well as other chemotactic mediators. In support of the latter possibility, our preliminary studies have shown that other IFN- $\gamma$-inducible chemokines (IP-10 and ITAC) are up-regulated in this cardiac allograft model and may participate in the recruitment of CXCR3 + T lymphocytes (JJ Yun, unpublished data).

Development of CAV in this MHC class II mismatched model is strictly dependent on CD4 lymphocytes and is further accentuated by CD8 lymphocytes. Our previous studies have shown that CD8 lymphocytes are activated by MHC class II disparate antigens (likely via cross priming) both in vivo and in vitro. ${ }^{4,18}$ Treatment of recipient mice with anti-MIG/CXCL9 serum markedly reduced the number of graft-infiltrating CD4 lymphocytes. However, we could not detect a significant change in the number of CD8 lymphocytes when the donor hearts were examined at day 24 after transplantation. Serial analysis in this model is needed to better define the effect of MIG/CXCL9 neutralization on $T$ lymphocyte subset trafficking. The lack of change in CD8 lymphocyte infiltration may be limited to day 24 after transplantation, as CD8 lymphocytes are known to express CXCR3 and be recruited to sites of inflammation. ${ }^{19}$ Furthermore, absence of CXCR3 or neutralization of MIG/CXCL9 in other models have resulted in reduction of both CD4 and CD8 Iymphocyte infiltration, when examined serially. ${ }^{12,20}$

In addition to reduction in CD4-lymphocyte recruitment, neutralizing MIG/CXCL9 may also directly affect the priming of recipient lymphocytes. To address this issue, we have performed intracellular cytokine assays on graft infiltrating lymphocytes from recipient mice treated with anti-MIG/CXCL9 serum and control serum. CD4 and CD8 lymphocytes from the donor hearts from anti-MIG/CXCL9 treated recipients produced Th-1 cytokines (IL-2, IFN- $\gamma$ ), but at a reduced rate when compared to the control group, suggesting that the attenuation of intimal proliferation in recipients treated with anti-MIG/ CXCL9 serum is, in part, because of inhibition of T lymphocyte recruitment.

We adapted the technique of intracellular cytokine staining to directly show that graft-infiltrating MOMA-2+ macrophages, but not CD4/CD8 lymphocytes, or natural killer cells produced MIG/CXCL9 in this model. It is important to note that this methodology aims to identify the recipient-derived graft-infiltrating cells that produce MIG/ CXCL9. MIG/CXCL9 is a known byproduct of monocytes/ macrophages activated with IFN- $\gamma .{ }^{21}$ Donor-derived cells 
such as endothelial cells have also been shown to be capable of producing MIG/CXCL9 in vitro on interaction with alloantigen-primed CD8 lymphocytes. ${ }^{22}$

Although CD4 lymphocytes did not produce MIG/ CXCL9, they were required for sustained MIG/CXCL9 production in this model. It is possible that CD4 lymphocytes regulate MIG/CXCL9 production via sustained secretion of cytokines (ie, IFN- $\gamma$ ) and/or via recruitment of other cells that participate in IFN- $\gamma$ and/or MIG/CXCL9 production. The findings of this study support a reciprocal regulatory relationship between MIG/CXCL9 and CD4 lymphocytes. We present evidence that MIG/CXCL9 is critical in CD4 lymphocyte recruitment in CAV (neutralization of MIG/CXCL9 decreased CD4 lymphocyte infiltration and intimal lesion development), and moreover that CD4 lymphocytes modulate MIG/CXCL9 production in this model (in absence of CD4 lymphocytes, intragraft MIG/CXCL9 level was low and transient).

It is interesting to note that a transient rise in MIG/ CXCL9, as was noted in the donor hearts in CD4 KO recipients, was not associated with intimal lesion development. Sustained MIG/CXCL9 production was necessary for persistent intragraft $\mathrm{T}$ lymphocyte recruitment, which is a known hallmark of chronic rejection. This observation is of clinical relevance in that transient and limited up-regulation of MIG/CXCL9 because of acute cellular rejection or other factors is insufficient to lead to chronic rejection. Repeated episodes of acute rejection may lead to recurrent up-regulation of MIG/CXCL9, and has been shown to be a risk factor for chronic rejection. ${ }^{2}$

In conclusion, the findings of this study suggest an important role for sustained MIG/CXCL9 level in T lymphocyte recruitment and CAV development. Neutralization of MIG/CXCL9 attenuates but does not abrogate CAV, suggesting that strategies that block multiple chemokines and/or chemokine receptors may be necessary in controlling chronic rejection.

\section{References}

1. Weis M, Von Scheidt W: Cardiac allograft vasculopathy. Circulation 1997, 96:2069-2077

2. Tullius SG, Tilney NL: Both alloantigen-dependent and independent factors influence chronic allograft rejection. Transplantation 1995 , 59:313-318

3. Shi C, Lee WS, He Q, Zhang D, Fletcher DL, Newell JB, Haber E: Immunologic basis of transplant-associated arteriosclerosis. Proc Natl Acad Sci USA 1996, 93:4051-4056

4. Fischbein MP, Yun J, Laks H, Irie Y, Fishbein MC, Espejo M, Bonavida B, Ardehali A: CD8+ lymphocytes augment chronic rejection in a MHC class II mismatched model. Transplantation 2001, 71:11461153

5. Gerard C, Rollins B: Chemokines and disease. Nat Immunol 2001, 2:108-115

6. Kondo TA, Novick C, Toma H, Fairchild RL: Induction of chemokine gene expression during allogeneic skin graft rejection. Transplantation 1996, 61:1750-1757

7. Yun J, Fischbein MF, Laks H, Fishbein MC, Espejo M, Ebrahimi K, Irie Y, Berliner JA, Ardehali A: Early and late chemokine production correlates with cellular recruitment in cardiac allograft vasculopathy. Transplantation 2000, 69:2515-2524

8. Bradley LM, Asensio VC, Schoitz LK, Harbertson J, Krahl T, Patstone G, Woolf N, Campbell IL, Savertnick N: Islet-specific Th1 but not Th2, cells secrete multiple chemokines and promote rapid induction of autoimmune diabetes. J Immunol 1999, 162:2511-2520

9. Shields PL, Morland CM, Salmon M, Qin S, Hubscher SG, Adams DH: Chemokine and chemokine receptor interactions provide a mechanism for selective $\mathrm{T}$ cell recruitment to specific liver compartments within hepatitis C-infected liver. J Immunol 1999, 163:6236-6243

10. Liu MT, Armstrong D, Hamilton TA, Lane TE: Expression of Mig (monokine induced by interferon- $\gamma$ ) is important in $T$ lymphocyte recruitment and host defense following viral infection of the central nervous system. J Immunol 2001, 166:1790-1795

11. Koga S, Auerbach MB, Engeman TM, Novick AC, Toma H, Fairchild $\mathrm{RL}$ : T cell infiltration into class II MHC-disparate allografts and acute rejection is dependent on the IFN-gamma-induced chemokine Mig. $\mathrm{J}$ Immunol 1999, 163:4878-4885

12. Miura M, Morita K, Kobayashi $\mathrm{H}$, Hamilton TA, Burdick MD, Strieter RM, Fairchild RL: Monokine induced by IFN-gamma is a dominant factor directing $T$ cells to murine cardiac allografts during acute rejection. J Immunol 2001, 167:3494-3504

13. Corry R, Winn H, Russell P: Primarily vascularized allografts of hearts in mice. The role of $\mathrm{H}-2 \mathrm{D}, \mathrm{H}-2 \mathrm{~K}$, and non- $\mathrm{H}-2$ antigens in rejection. Transplantation 1973, 16:343-350

14. Belperio JA, Keane MP, Burdick MD, Lynch III JP, Xue YY, Berlin A, Ross DJ, Kunkel SL, Charo IF, Strieter RM: Critical role for the chemokine MCP-1/CCR2 in the pathogenesis of bronchiolitis obliterans syndrome in lung transplant recipients. J Clin Invest 2001, 108:547554

15. Yun J, Fischbein MP, Laks H, Irie Y, Espejo M, Fishbein MC, Berliner $J$, Ardehali A: RANTES production during development of cardiac allograft vasculopathy. Transplantation 2001, 71:1649-1656

16. Fischbein MP, Ardehali A, Yun J, Schoenberger S, Laks H, Irie Y, Dempsey P, Cheng G, Fishbein MC, Bonavida B: CD40 signaling replaces $\mathrm{CD} 4+$ lymphocytes and its blocking prevents chronic rejection of heart transplants. J Immunol 2000, 165:7316-7322

17. Ring GH, Saleem S, Dai Z, Hassan AT, Zonieczny BT, Baddoura FK, Lakkis FG: Interferon-gamma is necessary for initiating the acute rejection of major histocompatibility complex class $\|$-disparate skin allografts. Transplantation 1999, 67:1362-1365

18. Fischbein MP, Yun J, Laks H, Irie Y, Fishbein MC, Bonavida B, Ardehali A: Role of CD8+ lymphocytes in chronic rejection of transplanted hearts. J Thorac Cardiovasc Surgery 2002, 123:803-809

19. Agostini C, Facco M, Siviero M, Carollo D, Galvan S, Cattelan A, Zambello R, Trentin L, Semenzato G: CXC chemokines IP-10 and Mig expression directs migration of pulmonary CD8+/CXCR3 $+\mathrm{T}$ cells in the lungs of patients with HIV infection and T-cell alveolitis. Am J Respir Crit Care Med 2000, 162:1466-1473

20. Hancock WW, Lu B, Gao W, Csizmadia V, Faia K, King JA, Smiley ST, Ling M, Gerard NP, Gerard C: Requirement of the chemokine receptor CXCR3 for acute allograft rejection. J Exp Med 2000, 192:15151520

21. Kunz MA, Toksoy M, Goebeler M, Engelhardt E, Brocker E, Gillitzer R: Strong expression of the lymphoattractant $\mathrm{C}-\mathrm{X}-\mathrm{C}$ chemokine Mig is associated with heavy infiltration of $\mathrm{T}$ cells in human malignant melanoma. J Pathol 1999, 189:552-558

22. Kapoor A, Morita K, Engeman TM, Koga S, Vapnek EM, Hobart MG, Fairchild RL: Early expression of interferon- $\gamma$ inducible protein 10 and monokine induced by interferon- $\gamma$ in cardiac allografts is mediated by CD8+ T cells. Transplantation 2000, 69:1147-1155 